University of Nebraska - Lincoln

DigitalCommons@University of Nebraska - Lincoln

Xia Hong Publications

Research Papers in Physics and Astronomy

2014

\title{
Nonlinear transport in nanoscale phase separated colossal magnetoresistive oxide thin films
}

\author{
V.R. Singh \\ L. Zhang \\ A. K. Rajapitamahuni \\ N. Devires \\ X. Hong
}

Follow this and additional works at: https://digitalcommons.unl.edu/physicshong

Part of the Atomic, Molecular and Optical Physics Commons, and the Engineering Physics Commons

This Article is brought to you for free and open access by the Research Papers in Physics and Astronomy at DigitalCommons@University of Nebraska Lincoln. It has been accepted for inclusion in Xia Hong Publications by an authorized administrator of DigitalCommons@University of Nebraska Lincoln. 


\title{
Nonlinear transport in nanoscale phase separated colossal magnetoresistive oxide thin films
}

\author{
V. R. Singh, ${ }^{1}$ L. Zhang, ${ }^{1}$ A. K. Rajapitamahuni, ${ }^{1}$ N. Devries,${ }^{1}$ and X. Hong ${ }^{1,2, a)}$ \\ ${ }^{1}$ Department of Physics and Astronomy, University of Nebraska-Lincoln, Nebraska 68588, USA \\ ${ }^{2}$ Nebraska Center for Materials and Nanoscience, University of Nebraska-Lincoln, Nebraska 68588, USA
}

(Received 17 May 2014; accepted 8 July 2014; published online 17 July 2014)

\begin{abstract}
We report a study of the $I-V$ characteristics of 2.5-5.4 nm epitaxial $\mathrm{La}_{1-x} \mathrm{Sr}_{x} \mathrm{MnO}_{3}(x=0.33$ and 0.5 ) and $\mathrm{La}_{0.7} \mathrm{Ca}_{0.3} \mathrm{MnO}_{3}$ thin films. While $\mathrm{La}_{0.67} \mathrm{Sr}_{0.33} \mathrm{MnO}_{3}$ films exhibit linear conduction over the entire temperature and magnetic field ranges investigated, we observe a strong correlation between the linearity of the $I-V$ relation and the metal-insulator transition in highly phase separated $\mathrm{La}_{0.5} \mathrm{Sr}_{0.5} \mathrm{MnO}_{3}$ and $\mathrm{La}_{0.7} \mathrm{Ca}_{0.3} \mathrm{MnO}_{3}$ films. Linear $I-V$ behavior has been observed in the high temperature paramagnetic insulating phase, and an additional current term proportional to $V^{\alpha}$ $(\alpha=1.5-2.8)$ starts to develop below the metal-insulator transition temperature $T_{\mathrm{MI}}$, with the onset temperature of the nonlinearity increasing in magnetic field as $T_{\mathrm{MI}}$ increases. The exponent $\alpha$ increases with decreasing temperature and increasing magnetic field and is significantly enhanced in ultrathin films with thicknesses close to that of the electrically dead layer. We attribute the origin of the nonlinearity to transport through the nanoscale coexisting metallic and insulating regions. Our results suggest that phase separation is not fully quenched even at low temperatures and high magnetic fields. (C) 2014 AIP Publishing LLC. [http://dx.doi.org/10.1063/1.4890605]
\end{abstract}

\section{INTRODUCTION}

Correlated oxides such as colossal magnetoresistive (CMR) manganites exhibit diverse electronic and magnetic properties that are tunable via carrier density, magnetic field, and strain, ${ }^{1,2}$ which make them a promising material platform for the development of nanoscale, multifunctional electronic and spintronic devices. ${ }^{3-11}$ For example, manganite thin films have been used to build $p-n$ junction and Schottky junction devices that exhibit rectified $I-V$ characteristics. ${ }^{4,10}$ To facilitate the rational design and optimize the performance of such devices, it is important to understand the intrinsic $I-V$ relation of manganite thin films. In previous studies, both linear and nonlinear $I-V$ behaviors have been reported in CMR manganites. ${ }^{12-20}$ The nonlinear conduction has been attributed to the melting of the charge ordered states via current, ${ }^{12-14}$ transport through grain boundaries, ${ }^{15-18}$ magnetic polarons, ${ }^{19}$ and nanoscale phase separation. ${ }^{13,20}$

In this work, we examine the critical role of nanoscale phase separation in nonlinear conduction of epitaxial manganite thin films. We have investigated the $I-V$ characteristics of $2.5-5.4 \mathrm{~nm}$ epitaxial single crystalline $\mathrm{La}_{1-x} \mathrm{Sr}_{x} \mathrm{MnO}_{3}$ (LSMO, $x=0.33$ and 0.5 ) and $\mathrm{La}_{1-x} \mathrm{Ca}_{x} \mathrm{MnO}_{3}$ (LCMO, $x=0.3)$ thin films. The $\mathrm{La}_{0.67} \mathrm{Sr}_{0.33} \mathrm{MnO}_{3}$ films exhibit linear conduction over the entire temperature and magnetic field ranges investigated. In the highly phase separated LCMO and $\mathrm{La}_{0.5} \mathrm{Sr}_{0.5} \mathrm{MnO}_{3}$ films, we observe a strong correlation between the linearity of the $I-V$ relation and the metalinsulator transition (MIT). Linear $I-V$ dependence has been observed in the high temperature paramagnetic insulating phase, and an additional term proportional to $V^{\alpha}(\alpha=1.5-2.8)$ starts to develop in the current below the metal-insulator

\footnotetext{
a) Author to whom correspondence should be addressed. Electronic mail: xhong2@unl.edu
}

transition temperature $T_{\mathrm{MI}}$. The onset temperature of the nonlinearity increases in magnetic field as $T_{\mathrm{MI}}$ increases. The values of the exponent $\alpha$ increase monotonically with decreasing temperature and increasing magnetic field, and the highest exponent has been observed in $\mathrm{La}_{0.5} \mathrm{Sr}_{0.5} \mathrm{MnO}_{3}$ films with thicknesses close to that of the electrically dead layer. We attribute the nonlinearity of the $I-V$ relation to transport through the nanoscale coexisting metallic and insulating regions in these phase separated manganite thin films.

\section{SAMPLE PREPARATION AND EXPERIMENTAL DETAILS}

We have deposited epitaxial LSMO and LCMO films on (001) $\mathrm{SrTiO}_{3}$ (STO) and (110) $\mathrm{NdGaO}_{3}$ (NGO) substrates using off-axis radio frequency magnetron sputtering. The films are grown at $650{ }^{\circ} \mathrm{C}$, with $150 \mathrm{mTorr}$ process gas composed of $\mathrm{Ar}$ and $\mathrm{O}_{2}$ with the ratio of 2:1. These films are (001) oriented, with no impurity phase observed in X-ray diffraction and a surface roughness of $\sim 2 \AA$ (Fig. S1 in the supplementary material). ${ }^{21}$ We then pattern the samples into Hall bar and two-point devices with channel widths of 5-20 $\mu \mathrm{m}$ using photolithography followed by the deposition of $30 \mathrm{~nm}$ Au electrodes. The $I-V$ and magnetotransport measurements have been taken in both two-point and four-point device geometries. The studies have been carried out in a Quantum Design Physical Property Measurement System (PPMS) using external sourcemeters (Keithley 2400) and low frequency lock-in techniques.

\section{EXPERIMENTAL RESULTS}

Figure 1(a) shows the temperature dependence of the resistivity for a $5.4 \mathrm{~nm} \mathrm{La} \mathrm{La}_{0.67} \mathrm{Sr}_{0.33} \mathrm{MnO}_{3}$ film grown on STO. At zero magnetic field, the film exhibits a transition from high temperature insulating behavior to low temperature 

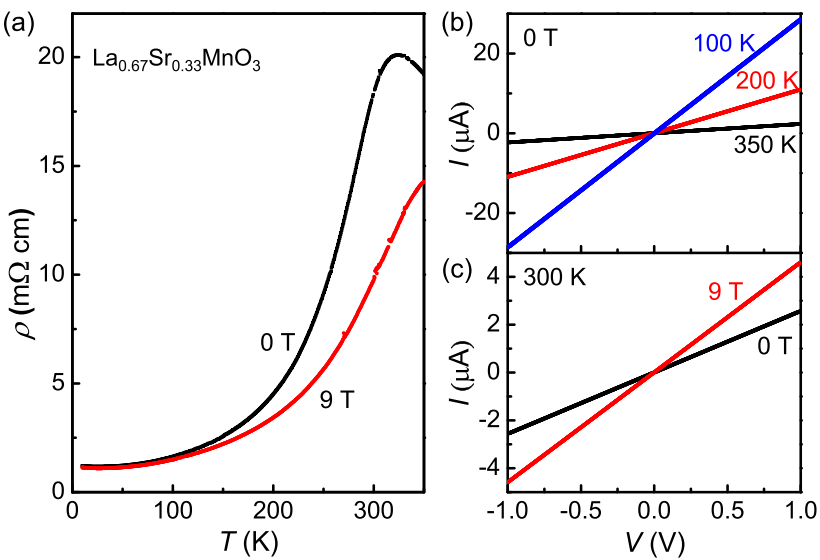

FIG. 1. Transport for a $5.4 \mathrm{~nm} \mathrm{La}_{0.67} \mathrm{Sr}_{0.33} \mathrm{MnO}_{3}$ film on (001) STO. (a) Resistivity as a function of temperature at $0 \mathrm{~T}$ and $9 \mathrm{~T}$. (b) Current as a function of bias voltage at $100 \mathrm{~K}, 200 \mathrm{~K}$, and $350 \mathrm{~K}$. (c) $I-V$ at $300 \mathrm{~K}$, and $0 \mathrm{~T}$ and $9 \mathrm{~T}$.

metallic behavior at $T_{\mathrm{MI}} \sim 325 \mathrm{~K}$. As LSMO is a widebandwidth system, its magnetoresistance (MR) is relatively small, with $R(0 \mathrm{~T}) / R(9 \mathrm{~T}) \sim 1.6$ at $T_{\mathrm{MI}}$. Figure $1(\mathrm{~b})$ shows the bias voltage dependence of current at temperatures above $(350 \mathrm{~K})$ and below the $T_{\mathrm{MI}}(200 \mathrm{~K}$ and $100 \mathrm{~K})$. We observe linear $I-V$ characteristics over the entire temperature range investigated $(10 \mathrm{~K}-350 \mathrm{~K})$. The linearity is preserved in magnetic fields up to $9 \mathrm{~T}$ (Fig. 1(c)).

We have then studied an intermediate bandwidth system, $\mathrm{La}_{0.7} \mathrm{Ca}_{0.3} \mathrm{MnO}_{3}$, which exhibits a lower $T_{\mathrm{MI}}$ and more pronounced MR compared with those in LSMO of similar composition. Figure 2(a) shows $\rho(T)$ for a $4.2 \mathrm{~nm}$ LCMO film, which shows a $T_{\mathrm{MI}} \sim 145 \mathrm{~K}$ and $R(0 \mathrm{~T}) / R(9 \mathrm{~T}) \sim 95$ at $T_{\mathrm{MI}}$. At $T>T_{\mathrm{MI}}$, the film exhibits linear conduction, similar to what is observed in $\mathrm{La}_{0.67} \mathrm{Sr}_{0.33} \mathrm{MnO}_{3}$. As the sample is
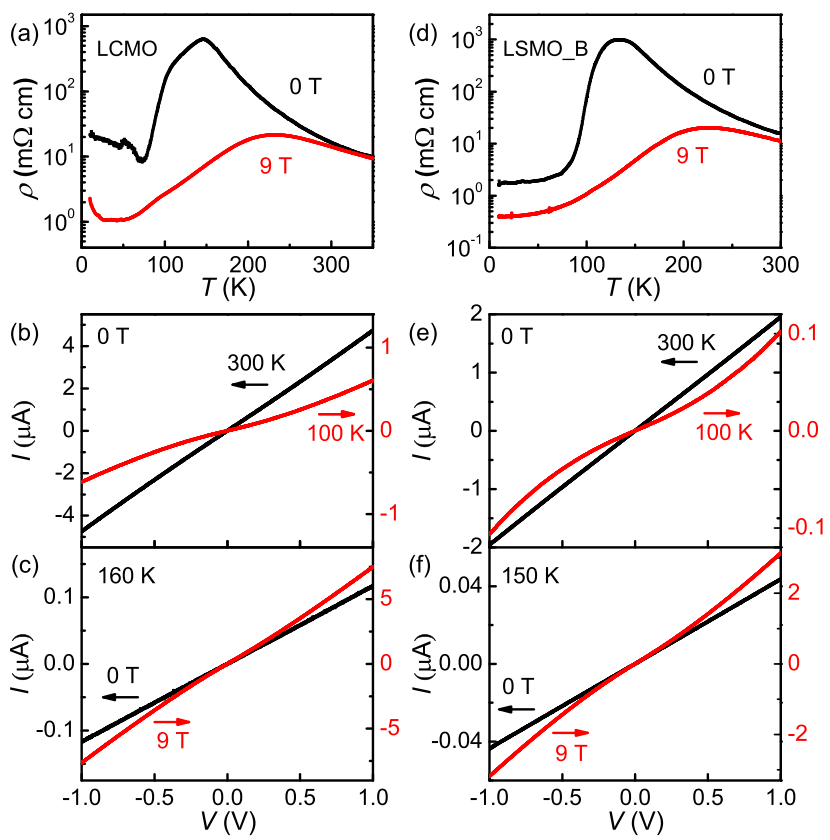

FIG. 2. (a)-(c) Transport for a $4.2 \mathrm{~nm}$ LCMO film on (001) STO. (a) $\rho$ (T) at $0 \mathrm{~T}$ and $9 \mathrm{~T}$. (b) $I-V$ at $100 \mathrm{~K}$ and $300 \mathrm{~K}$. (c) $I-V$ at $160 \mathrm{~K}$, and $0 \mathrm{~T}$ and $9 \mathrm{~T}$. (d)-(f) Transport for a $3.2 \mathrm{~nm} \mathrm{La}{ }_{0.5} \mathrm{Sr}_{0.5} \mathrm{MnO}_{3}$ film on (110) NGO (sample LSMO_B). (d) $\rho(T)$ at $0 \mathrm{~T}$ and $9 \mathrm{~T}$. (e) $I-V$ at $100 \mathrm{~K}$ and $300 \mathrm{~K}$. (f) $I-V$ at $150 \mathrm{~K}$, and $0 \mathrm{~T}$ and $9 \mathrm{~T}$. cooled down below $T_{\text {MI }}$, however, a nonlinear component of $V$-dependence starts to develop in the current (Fig. 2(b)). This onset of nonlinearity correlates strongly with the MIT. As shown in Fig. 2(a), the LCMO film is in the insulating phase at $160 \mathrm{~K}$, but becomes metallic in a magnetic field of $9 \mathrm{~T}$ as $T_{\mathrm{MI}}$ shifts to $232 \mathrm{~K}$. Interestingly, the $I-V$ relation at $160 \mathrm{~K}$ also changes from linear at $0 \mathrm{~T}$ to nonlinear as the film becomes metallic (Fig. 2(c)).

To confirm that the nonlinearity is intrinsic to the LCMO sample rather than due to extrinsic effects such as Joule heating and resistance switching, we have calculated the power density $p=j^{2} \rho$, where $j$ is the current density, of different samples with the same device geometry and at the same temperature (see supplementary material for a detailed discussion). ${ }^{21}$ Our calculation shows that the maximum power density in the LCMO and $\mathrm{La}_{0.67} \mathrm{Sr}_{0.33} \mathrm{MnO}_{3}$ samples are comparable at low temperatures, while the nonlinear $I-V$ has only been observed in the LCMO sample. The power density in our sample is also three orders of magnitude lower than the critical values reported for Joule heating in microstructured LCMO thin films. ${ }^{22}$ The observed $I-V$ curve is continuous and fully reproducible as we sweep voltage in different directions and with different speeds, while Joule heating or current induced resistance switching often leads to a sudden softening or even an abrupt change in the current accompanied with hysteresis behavior. ${ }^{12,22}$ More important, as shown in Fig. 2(c), LCMO exhibits a super-linear $I-V$ at $9 \mathrm{~T}$, suggesting higher conductance at higher electric field. This is opposite to what is expected from Joule heating, which should give rise to a sub-linear $I-V$ as the sample is in the metallic phase at $160 \mathrm{~K}$ and $9 \mathrm{~T}$. We thus conclude that the nonlinearity in $I-V$ is intrinsic to LCMO thin films.

As we are working with ultrathin epitaxial films, one possible source for the nonlinear $I-V$ behavior is parallel conduction through an electrically dead layer. The existence of an electrically dead interfacial layer has been widely observed in epitaxial manganite thin films and has been attributed to structural or chemical modification at the surface and interface. ${ }^{6,23,24}$ We have examined the thickness dependence of the transport properties of our $\mathrm{La}_{0.67} \mathrm{Sr}_{0.33} \mathrm{MnO}_{3}$ films and found the critical thickness for the electrically dead layer to be $3.2 \mathrm{~nm}$. We then worked with a $2.5 \mathrm{~nm}$ thick $\mathrm{La}_{0.67} \mathrm{Sr}_{0.33} \mathrm{MnO}_{3}$ film, which is insulating at all temperatures. The film exhibits linear $I-V$ characteristics in the entire temperature and magnetic field ranges investigated (supplementary material), ${ }^{21}$ which suggests that the existence of the electrically dead layer is not the source of the nonlinearity.

The key difference between the $\mathrm{La}_{0.67} \mathrm{Sr}_{0.33} \mathrm{MnO}_{3}$ and LCMO samples is LCMO is highly phase separated, with coexisting nanoscale metallic and insulating regions. ${ }^{2}$ Transport through such a network of metallic regions is percolative, ${ }^{25}$ and hopping or tunneling through nanoscale insulating regions can give rise to nonlinear $I-V$ characteristics. ${ }^{26}$ To identify the role of phase separation in the nonlinear transport, we have investigated another LSMO system with $x=0.5$. The ground state of the half doped LSMO is close to the phase boundary between the hole-doped ferromagnetic phase and the electron-doped antiferromagnetic/charge ordered phase, which naturally leads to coexistence of competing phases at 
the nanoscale., ${ }^{2,27}$ We have measured two $\mathrm{La}_{0.5} \mathrm{Sr}_{0.5} \mathrm{MnO}_{3}$ films grown on NGO substrates. The $5 \mathrm{~nm}$ sample (denoted as LSMO_A) shows a $T_{\mathrm{MI}}$ of $250 \mathrm{~K}$, close to the values observed in thick films. The $3.2 \mathrm{~nm}$ sample (denoted as LSMO_B) shows a significantly lower $T_{\mathrm{MI}}$ of $135 \mathrm{~K}$ (Fig. 2(d)), as the film thickness is close to the electrically dead layer thickness for this composition $(\sim 3 \mathrm{~nm})$. Its large MR of $R(0 \mathrm{~T}) / R(9 \mathrm{~T}) \sim 340$ at $T_{\mathrm{MI}}$ is a direct manifestation of the presence of enhanced phase separation. The $I-V$ relations of both $\mathrm{La}_{0.5} \mathrm{Sr}_{0.5} \mathrm{MnO}_{3}$ films closely resemble that of the LCMO films, with linear $I-V$ at high temperature and nonlinear $I-V$ below $T_{\mathrm{MI}}$, which can be tuned by a magnetic field. Figures 2(e) and 2(f) show clearly the effects of temperature and magnetic field on the $I-V$ behavior for sample LSMO_B, respectively. We observe linear $I-V$ at $300 \mathrm{~K}$ (above $T_{\mathrm{MI}}$ ) and nonlinear $I-V$ at $100 \mathrm{~K}$ (below $T_{\mathrm{MI}}$ ). At $150 \mathrm{~K}$, the conduction of the sample is tuned from linear at $0 \mathrm{~T}$ to nonlinear in magnetic field of $9 \mathrm{~T}$, while $T_{\mathrm{MI}}$ increases from $135 \mathrm{~K}$ to $226 \mathrm{~K}$.

In both materials systems, the $I-V$ relation can be well described by the following expression:

$$
I=G_{0} V+G_{1} V^{\alpha},
$$

where the nonlinear exponent $\alpha$ is a function of temperature and magnetic field. Figure 3(a) shows the temperature dependence of the exponent $\alpha$ taken on the LCMO sample. At zero magnetic field, $\alpha$ changes from about 1.6 at $130 \mathrm{~K}$ to 2.0 at $10 \mathrm{~K}$, showing a monotonic increase with decreasing
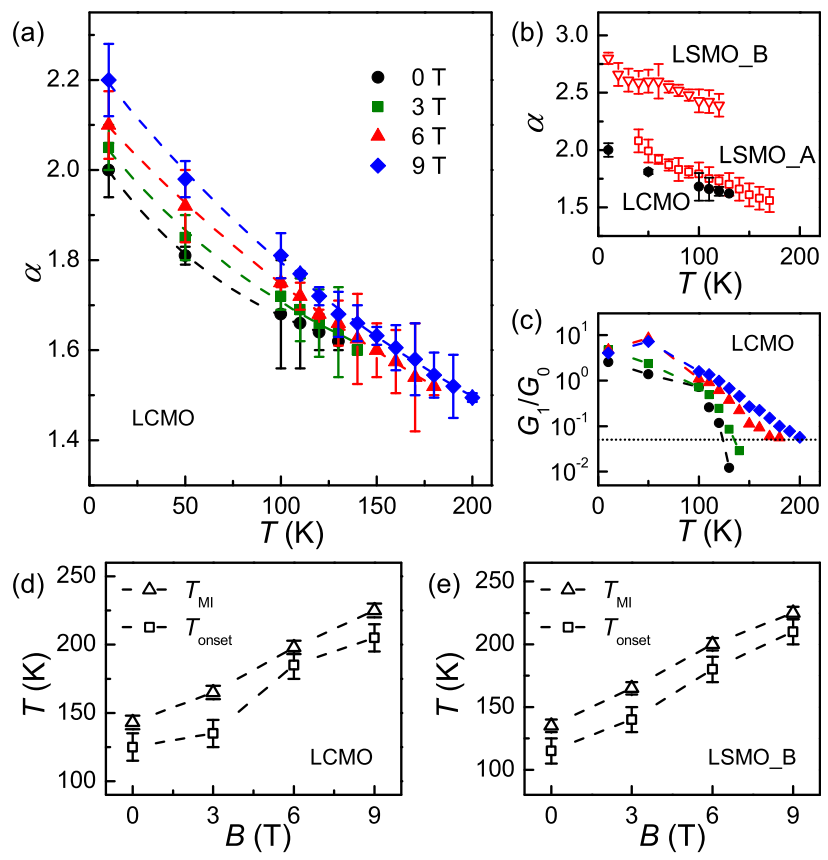

FIG. 3. (a) Temperature dependence of the nonlinear exponent $\alpha$ at magnetic fields of $0 \mathrm{~T}$ (black circles), $3 \mathrm{~T}$ (green squares), $6 \mathrm{~T}$ (red triangles), and $9 \mathrm{~T}$ (blue diamonds) for the $4.2 \mathrm{~nm}$ LCMO sample shown in Figs. 2(a)-2(c). (b) Temperature dependence of $\alpha$ at zero magnetic field for the LCMO sample (solid circles), sample LSMO_A (open squares), and sample LSMO_B (open triangles). (c) Temperature dependence of $G_{1} / G_{0}$ at magnetic fields of $0 \mathrm{~T}$ (black circles), $3 \mathrm{~T}$ (green squares), $6 \mathrm{~T}$ (red triangles), and $9 \mathrm{~T}$ (blue diamonds) for the LCMO sample. The dotted line marks $G_{1} / G_{0}=0.05$. (d) Magnetic field dependence of $T_{\mathrm{MI}}$ (open triangles) and $T_{\text {onset }}$ (open squares) for the LCMO sample. (e) Magnetic field dependence of $T_{\mathrm{MI}}$ (open triangles) and $T_{\text {onset }}$ (open squares) for sample LSMO_B. temperature. In a magnetic field, the nonlinearity emerges at higher temperature and corresponds to a larger $\alpha$, reaching $\sim 2.2$ at $10 \mathrm{~K}$ and $9 \mathrm{~T}$. The exponents observed on the halfdoped LSMO samples exhibit qualitatively similar temperature dependences (Fig. 3(b)). While sample LSMO_A shows nonlinear exponent values comparable with those observed in the LCMO sample, the exponents are significantly enhanced in LSMO_B. Note that the thickness of LSMO_B is close to that of the electrically dead layer, with a significantly suppressed $T_{\mathrm{MI}}$ and enhanced MR, which suggests an enhanced level of phase separation.

We then use the ratio between $G_{1}$ and $G_{0}$ (Eq. (1)) to quantify the onset temperature of the nonlinear $I-V$, and study how it is related to the $T_{\mathrm{MI}}$. Figure 3(c) shows the temperature dependence of $G_{1} / G_{0}$ for the LCMO sample at different magnetic fields. We define the $I-V$ curve as linear when $G_{1} / G_{0}<0.05$, which is close to experimental error, and extract the corresponding onset temperature $T_{\text {onset }}$. The temperature dependences of both $T_{\mathrm{MI}}$ and $T_{\text {onset }}$ for LCMO and LSMO_B are shown in Figs. 3(d) and 3(e), respectively. It is clear that for both LCMO and half doped LSMO, $T_{\text {onset }}$ increases with $T_{\mathrm{MI}}$ in magnetic fields, confirming the correlation between the MIT and the onset of nonlinearity.

\section{DISCUSSIONS}

In previous studies, nonlinear conduction has been observed in a variety of manganite systems. Besides Joule heating ${ }^{22}$ and current induced melting of the charge ordered states, ${ }^{12-14}$ which we have already ruled out, the presence of magnetic polarons, ${ }^{19}$ tunneling through grain boundaries, ${ }^{15-18}$ and nanoscale phase separation ${ }^{13,20}$ have also been proposed as possible sources for the nonlinear conduction. For example, it has been suggested that magnetic polarons are responsible for the nonlinear high frequency electrical response in LCMO probed by third harmonic resistance. ${ }^{19}$ However, such nonlinearity has only been observed above $T_{\mathrm{MI}}$ and showed significant enhancement at temperatures close to the transition temperature, where a high density of magnetic polarons are expected to be activated. ${ }^{28}$ In addition, the magnetic polaron induced effect has been found to be suppressed in magnetic field, consistent with the notion that magnetic polarons should collapse in magnetic fields. ${ }^{29}$ In sharp contrast, the nonlinear behavior in DC $I-V$ relation reported here is only observed at $T<T_{\mathrm{MI}}$ and becomes more pronounced at lower temperatures. Moreover, the nonlinearity is enhanced at high magnetic field. We thus rule out magnetic polaron as the origin of the nonlinear $I-V$.

Another scenario based on tunneling through grain boundaries has been proposed to account for the nonlinear conduction observed in thin manganite films grown on bicrystal substrates ${ }^{15-17}$ and polycrystalline thin films. ${ }^{18,30,31}$ It has been shown that there is a large chemical potential difference $(\Delta \mu)$, on the order of tenths of the conduction band width, between the insulating and ferromagnetic metallic (FM) phases in doped manganites. ${ }^{32}$ As a result, significant band bending forms at the interface between the FM phase and the insulating grain boundary for $T<T_{\mathrm{MI}}$, which is close to the magnetic Curie temperature $T_{\mathrm{C}}$. Transport through 
such local energy barriers leads to nonlinear $I-V$ behavior. As $\Delta \mu$ is proportional to the magnetization of the sample, we expect an enhanced energy barrier and reduced thermal activation at lower temperatures, which leads to enhanced nonlinearity in transport. ${ }^{17}$

The picture of nonlinear transport through local energy barriers due to the work function difference between different phases can naturally explain some key features of our observation. First, the nonlinear effect only emerges below $T_{\mathrm{MI}}$, as band bending occurs at the interface between the insulating and metallic regions. Second, the nonlinearity becomes more pronounced at lower temperatures due to enhanced band bending and suppression of thermal excitation related transport. Although our films are single crystalline and TEM images reveal no grain boundaries, it is important to note that phase separation can lead to coexisting nanoscale insulating regions in the FM phase. ${ }^{2}$ The nonlinear behavior is only observed in the highly phase separated LCMO and half doped LSMO, revealing clearly the critical role of phase separation. Third, this picture is also consistent with the observation that magnetic field enhances the nonlinear behavior. The presence of a magnetic field increases magnetization, which leads to larger band bending and higher energy barrier. It also reduces the total volume of the insulating phase, which effectively reduces the thickness of the insulating barrier for the charge carriers to tunnel through. It thus makes transport through tunneling more efficient. The low temperature exponent values $\alpha=2.0-2.8$ are close to the calculated value of $7 / 3$ for amorphous semiconductor thin films ${ }^{33}$ and the exponent of 2.47 reported in discontinuous 2D Au thin films close to a percolation threshold, ${ }^{26}$ suggesting the strong similarity between our study and transport through highly inhomogeneous, disordered systems. We thus attribute the origin of the nonlinearity to transport through nanoscale phase separated regions. ${ }^{13,20}$

This model also explains the different temperature dependences of the nonlinearity and the magnetoresistance. Since the CMR effect is due to the melting of the insulating phase in the presence of magnetic field, MR can be taken as a direct measure of the degree of phase separation in the system. It is known that the MR peaks at temperatures just below $T_{\mathrm{MI}}$, where a maximum level of phase separation is expected. The fact that our observation is enhanced at low temperatures and high magnetic fields is consistent with the picture that it is the work function difference between different phases that lead to nonlinear $I-V$, and the degree of the phase separation plays a minor role in determining the $I-V$ characteristics. An important implication of our results is even at low temperatures and a magnetic field of $9 \mathrm{~T}$, where the magnetoresistance becomes relatively weak, phase separation has not been fully quenched. This picture agrees well with previous X-ray scattering studies of the effect of magnetic field on charge/orbitally ordered nanoclusters in manganite. ${ }^{29}$

\section{CONCLUSIONS}

In conclusion, we have observed strong nonlinear conduction in highly phase separated LCMO and half-doped LSMO thin films. The nonlinearity closely correlates with the metal-insulator transition and can be enhanced by magnetic fields and in films with thicknesses close to that of the electrically dead layer. We attribute its origin to transport through energy barriers due to the interfacial work function difference between the nanoscale phase separated insulating and metallic regions. Our results also suggest that phase separation is not fully quenched even at low temperatures and high magnetic fields.

\section{ACKNOWLEDGMENTS}

We thank P. Dowben, S. Adenwalla, X. Xu, and H. J. Gardner for enlightening discussions and technical assistance. This research was performed in part in the Central Facilities of the Nebraska Center for Materials and Nanoscience, which was supported by the Nebraska Research Initiative. X.H. acknowledges support from NSF CAREER Grant No. DMR-1148783, NSF MRSEC Grant No. DMR-0820521, and the Center for NanoFerroic Devices (CNFD) and the Nanoelectronics Research Initiative (NRI).

${ }^{1}$ Colossal Magnetoresistive Oxides, edited by Y. Tokura (CRC Press, Amsterdam, Netherlands, 2000).

${ }^{2}$ E. Dagotto, T. Hotta, and A. Moreo, Phys. Rep. 344, 1 (2001).

${ }^{3}$ T. Wu et al., Phys. Rev. Lett. 86, 5998 (2001).

${ }^{4}$ H. Tanaka, J. Zhang, and T. Kawai, Phys. Rev. Lett. 88, 027204 (2002).

${ }^{5}$ X. Hong, A. Posadas, A. Lin, and C. H. Ahn, Phys. Rev. B 68, 134415 (2003).

${ }^{6}$ X. Hong, A. Posadas, and C. H. Ahn, Appl. Phys. Lett. 86, 142501 (2005). ${ }^{7}$ Y. Bason, L. Klein, J. B. Yau, X. Hong, J. Hoffman, and C. H. Ahn, J. Appl. Phys. 99, 08R701 (2006).

${ }^{8}$ R. Ramesh and D. G. Schlom, MRS Bull. 33, 1006 (2008).

${ }^{9}$ R. W. Li, H. B. Wang, X. W. Wang, X. Z. Yu, Y. Matsui, Z. H. Cheng, B. G. Shen, E. W. Plummer, and J. D. Zhang, Proc. Natl. Acad. Sci. U. S. A. 106, 14224 (2009).

${ }^{10}$ Y. Hikita and H. Y. Hwang, Complex Oxide Schottky Junctions (Springer, New York, 2010)

${ }^{11}$ J. Hoffman, X. Hong, and C. H. Ahn, Nanotechnology 22, 254014 (2011).

${ }^{12}$ A. Asamitsu, Y. Tomioka, H. Kuwahara, and Y. Tokura, Nature 388, 50 (1997).

${ }^{13}$ A. Guha, A. K. Raychaudhuri, A. R. Raju, and C. N. R. Rao, Phys. Rev. B 62, 5320 (2000).

${ }^{14}$ P. Padhan, W. Prellier, C. Simon, and R. C. Budhani, Phys. Rev. B 70, 134403 (2004).

${ }^{15}$ N. K. Todd, N. D. Mathur, S. P. Isaac, J. E. Evetts, and M. G. Blamire, J. Appl. Phys. 85, 7263 (1999).

${ }^{16}$ J. Klein, C. Hoffner, S. Uhlenbruck, L. Alff, B. Buchner, and R. Gross, Europhys. Lett. 47, 371 (1999).

${ }^{17}$ R. Gross et al., J. Magn. Magn. Mater. 211, 150 (2000).

${ }^{18}$ J. Philip and T. R. N. Kutty, Appl. Phys. Lett. 79, 209 (2001).

${ }^{19}$ V. Moshnyaga et al., Phys. Rev. B 79, 134413 (2009).

${ }^{20}$ F. X. Hu, J. Gao, and X. S. Wu, Phys. Rev. B 72, 064428 (2005).

${ }^{21}$ See supplementary material at http://dx.doi.org/10.1063/1.4890605 for the discussions on the film characterization, transport studies of a $3 \mathrm{~nm}$ LSMO film, and effect of Joule heating.

${ }^{22}$ C. Beekman, I. Komissarov, M. Hesselberth, and J. Aarts, Appl. Phys. Lett. 91, 062101 (2007).

${ }^{23}$ J. Z. Sun, D. W. Abraham, R. A. Rao, and C. B. Eom, Appl. Phys. Lett. 74, 3017 (1999)

${ }^{24}$ H. Dulli, P. A. Dowben, S. H. Liou, and E. W. Plummer, Phys. Rev. B 62, R14629 (2000).

${ }^{25}$ T. Z. Ward, J. D. Budai, Z. Gai, J. Z. Tischler, L. F. Yin, and J. Shen, Nat. Phys. 5, 885 (2009).

${ }^{26}$ Y. Gefen, W. H. Shih, R. B. Laibowitz, and J. M. Viggiano, Phys. Rev. Lett. 57, 3097 (1986).

${ }^{27}$ T. S. Santos, S. J. May, J. L. Robertson, and A. Bhattacharya, Phys. Rev. B 80, 155114 (2009).

${ }^{28}$ V. Kiryukhin, T. Y. Koo, H. Ishibashi, J. P. Hill, and S. W. Cheong, Phys. Rev. B 67, 064421 (2003). 
${ }^{29}$ T. Y. Koo, V. Kiryukhin, P. A. Sharma, J. P. Hill, and S. W. Cheong, Phys. Rev. B 64, 220405 (2001).

${ }^{30}$ C. Hofener, J. B. Philipp, J. Klein, L. Alff, A. Marx, B. Buchner, and R. Gross, Europhys. Lett. 50, 681 (2000).
${ }^{31}$ V. Markovich, G. Jung, S. I. Khartsev, M. I. Tsindlekht, A. M. Grishin, Y. Yuzhelevski, and G. Gorodetsky, J. Appl. Phys. 106, 043908 (2009).

${ }^{32}$ N. Furukawa, J. Phys. Soc. Jpn. 66, 2523 (1997).

${ }^{33}$ L. I. Glazman and K. A. Matveev, Sov. Phys. JETP 67, 1276 (1988). 